\title{
Antiproliferative and Anti-Invasive Effect of Piceatannol, a Polyphenol Present in Grapes and Wine, against Hepatoma AH109A Cells
}

\author{
Yuichiro Kita, ${ }^{1}$ Yutaka Miura, ${ }^{2}$ and Kazumi Yagasaki ${ }^{2}$ \\ ${ }^{1}$ Department of Applied Biological Chemistry, Graduate School of Agriculture, Tokyo University of Agriculture and Technology, Fuchu, \\ Tokyo 183-8509, Japan \\ ${ }^{2}$ Division of Applied Biological Chemistry, Institue of Agriculture, Tokyo University of Agriculture and Technology, Fuchu, \\ Tokyo 183-8509, Japan
}

Correspondence should be addressed to Kazumi Yagasaki, yagasaki@cc.tuat.ac.jp

Received 6 September 2011; Accepted 11 October 2011

Academic Editor: Masa-Aki Shibata

Copyright ( 2012 Yuichiro Kita et al. This is an open access article distributed under the Creative Commons Attribution License, which permits unrestricted use, distribution, and reproduction in any medium, provided the original work is properly cited.

\begin{abstract}
Piceatannol is a stilbenoid, a metabolite of resveratrol found in red wine. Piceatannol and sera from rats orally given piceatannol were found to dose-dependently suppress both the proliferation and invasion of AH109A hepatoma cells in culture. Its antiproliferative effect was based on cell cycle arrest at lower concentration $(25 \sim 50 \mu \mathrm{M})$ and on apoptosis induction at higher concentration $(100 \mu \mathrm{M})$. Piceatannol suppressed reactive oxygen species-potentiated invasive capacity by scavenging the intracellular reactive oxygen species. These results suggest that piceatannol, unlike resveratrol, has a potential to suppress the hepatoma proliferation by inducing cell cycle arrest and apoptosis induction. They also suggest that the antioxidative property of piceatannol, like resveratrol, may be involved in its anti-invasive action. Subsequently, piceatannol was found to suppress the growth of solid tumor and metastasis in hepatoma-bearing rats. Thus, piceatannol may be a useful anticancer natural product.
\end{abstract}

\section{Introduction}

Cancer cells have two biological properties, that is, endless proliferation and metastasis. Invasion is a most important and characteristic step in the complicated process of cancer cells [1]. The blockage of these biological events by drugs or factors from food and natural resources will prolong the life span of an affected host. Our previous research showed that green, oolong, and black tea extracts, the sera from rats orally given these teas, and related components inhibited the proliferation and invasion of a rat ascites hepatoma cell line of AH109A [2] through induction of apoptosis and cell cycle arrest [3] and antioxidative activity [4]. Lignans also suppressed both the proliferation and invasion of AH109A hepatoma cells in vitro, and hence solid tumor growth and metastasis of AH109A cells in vivo [5]. Resveratrol $\left(3,5,4^{\prime}\right.$-trihydroxy-trans-stilbene) is known to possess cancer chemopreventive activity and strong antioxidative activity [6]. Hsieh et al. reported that resveratrol showed a greater antiproliferative effect on highly invasive breast carcinoma cells (MDA-MB-435) than on minimally invasive cells
(MCF-7) [7]. However, our previous study showed that, in the hepatoma cells, resveratrol as such showed a weaker suppressive effect on the proliferation than on the invasion, and the sera from rats orally given resveratrol completely lost the antiproliferative activity in culture, although its anti-invasive activity was maintained $[8,9]$. These findings suggest that the effect of resveratrol on the proliferation may be dependent on cancer cell properties. Piceatannol $\left(3,4,3^{\prime}, 5^{\prime}\right.$-tetrahydroxytrans-stilbene), an analogue of resveratrol, has been reported to induce apoptosis and cell cycle arrest in human melanoma cell (SK-Mel-28) [10].

In the present study, we examined whether piceatannol or its in vivo metabolite(s) would have antiproliferative and anti-invasive effects against AH109A and their modes of actions.

\section{Materials and Methods}

2.1. Materials. Piceatannol was purchased from Sigma Chemical Co. (St. Louis, MO, USA). It was resolved in absolute ethanol (Wako Pure Chemical Industries, Ltd., Osaka, Japan) 
and the piceatannol solution was added to the medium at a final ethanol concentration of $0.2 \%$. Control medium contained $0.2 \%$ ethanol alone. All other reagents were of the best grade commercially available.

2.2. Culture of AH109A Hepatoma Cells. The animal experiments in this article were conducted in accordance with guidelines established by the Animal Care and Use Committee of Tokyo University of Agriculture and Technology and were approved by this committee. Male Donryu rats ( 4 weeks of age) were purchased from NRC Haruna (Gunma, Japan). AH109A cells were generously provided by the Cell Resource Center for Biomedical Research, Tohoku University, Sendai, Japan. AH109A cells were maintained in peritoneal cavity of male Donryu rats and isolated from accumulated ascites and then cultured in Eagle's minimum essential medium (MEM) (Nissui Pharmaceutical Co., Tokyo, Japan) containing 10\% calf serum (CS, JRH Biosciences, Lenaxa, KS, USA) (10\% CS/MEM). These cells were cultured for at least 2 weeks after isolation to eliminate contaminated macrophages and neutrophils and used for the assays described hereinafter.

2.3. In Vitro Proliferation and Invasion Assays. Effect of piceatannol on AH109A proliferation was examined by measuring the incorporation of [methyl- $\left.{ }^{3} \mathrm{H}\right]$ thymidine $(0.15 \mu \mathrm{Ci} /$ well, specific radioactivity; $20 \mathrm{Ci} / \mathrm{mmoL}$, PerkinElmer Life and Analytical Sciences, Boston, MA, USA) into DNA as described previously $[8,11]$. Effect of piceatannol on AH109A invasion was estimated by coculturing AH109A cells with rat mesentery-derived mesothelial cell (M-cell) monolayers, which were isolated and cultured from Donryu rats as described previously [12] with slight modifications [13]. Briefly, mesothelial cells (M-cells) were isolated from mesentery of male Donryu rats (6-8 weeks of age). After digestion by trypsin, $1.3-2.0 \times 10^{5} \mathrm{M}$-cells were plated in a $60 \mathrm{~mm}$ culture dish with $2 \mathrm{~mm}$ grids (Corning Incorporated, Corning, NY, USA) and cultured for 5-7 days to attain a confluent state in 10\% CS/MEM. Then, AH109A cells $(2.4 \times$ $10^{5}$ cells per dish) were applied on the monolayer of $\mathrm{M}$ cells in $10 \% \mathrm{CS} / \mathrm{MEM}$ with piceatannol for $24 \mathrm{~h}$. Invaded cells and colonies underneath $\mathrm{M}$-cells were counted with a phase-contrast microscope. The invasive activity of AH109A cells was expressed as the number of invaded cells and colonies $/ \mathrm{cm}^{2}$.

2.4. Ex Vivo Assay. Ex vivo assay was performed with piceatannol-loaded rat sera (RS). Piceatannol was suspended in a $0.3 \%$ carboxymethyl cellulose sodium salt (CMC, Wako Pure Chemical Industries, Osaka, Japan) aqueous solution at concentrations of $0.25,0.5$, and $1 \mathrm{mg} / \mathrm{mL}$, unless otherwise noted. Piceatannol suspension or $0.3 \%$ CMC alone (vehicle control) ( $1 \mathrm{~mL} / 100 \mathrm{~g}$ body weight) was intubated to male Donryu rats ( 5 weeks old) which had been fasted overnight, and blood was collected $2 \mathrm{~h}$ after oral administration as described previously $[8,13]$. Blood from rats given vehicle alone was also collected. The sera were prepared by centrifugation, sterilized by filtration, and added to the culture medium at a concentration of $10 \%$ instead of CS.
2.5. Flowcytometric Analyses of Cell Cycle Phases and AnnexinV-FITC Staining of Apoptotic Cells. For cell cycle analysis, $3.0 \times 10^{5}$ cells of AH109A per well were seeded in a 6-well plate in the medium containing various concentrations of piceatannol and cultured for $24 \mathrm{~h}$. Cells were collected and washed twice with sterilized, $\mathrm{Ca}^{2+}$ - and $\mathrm{Mg}^{2+}$-free, phosphate-buffered saline $[\mathrm{PBS}(-)]$. Thereafter, $500 \mu \mathrm{L}$ of propidium iodide (PI) solution containing $1 \mathrm{mg}$ of PI (Sigma Chemical Co., St. Louis, MO, USA) in $20 \mathrm{~mL}$ of $1 \%$ Triton X-100 (Sigma) and $0.1 \%$ of sodium citrate (Wako Pure Chemical Industries Ltd., Osaka, Japan) was added and cells were incubated for $30 \mathrm{~min}$ on ice. Cells at different cell cycle phases were then analyzed with a flow cytometer (EPICS ELITE EPS, Beckman-Coulter, Hialeah, FL, USA) as previously described [3]. The effect of piceatannol on apoptosis in AH109A cells was assessed using Annexin-V-FITC kit (IMMUNOTECH, Marseille, France) according to the manufacturer's instructions. Phosphatidylserine (PS), which can specifically bind to Annexin-V, is one of the phospholipids in the cell membrane and exists predominantly in inner leaflet of the cell membrane of normal cells. When apoptosis occurs, PS in the cell membrane immediately appears on the outer leaflet of the cell membrane. The cells with PS on their surface can thus be thought to be early apoptotic cells. Briefly, $5 \times 10^{5}$ cells of AH109A per well were seeded in a 6-well plate and cultured in the medium containing 0,50 , and $100 \mu \mathrm{M}$ piceatannol for $3 \mathrm{~h}$. At the end of culture, cells were labeled with Annexin-V-FITC and analyzed with a flow cytometer as described previously [14].

2.6. Pretreatment of AH109A Cells with Hydrogen Peroxide. AH109A cells were cultured for $4 \mathrm{~h}$ in the absence or presence of $10 \mu \mathrm{M}$ piceatannol with or without $25 \mu \mathrm{M}$ hydrogen peroxide $\left(\mathrm{H}_{2} \mathrm{O}_{2}\right.$, Wako Pure Chemical Industries). Hydrogen peroxide was employed as exogenous reactive oxygen species (ROS). AH109A cells were then washed once with 10\% CS/ MEM and seeded on the M-cell monolayer in 10\% CS/MEM without piceatannol and ROS. After cultured for $24 \mathrm{~h}$, invaded cells and colonies underneath M-cells were counted with a phase-contrast microscope as described previously.

2.7. Flowcytometric Analysis of Intracellular Peroxide in AH109A Cells. Intracellular peroxide levels in AH109A cells were assessed by flow cytometric analysis using a fluorometric probe $\left(2^{\prime}, 7^{\prime}\right.$-dichlorofluorescin diacetate; DCFH-DA, Molecular Probes, Eugene, OR) [15] with a flow cytometer as described previously [16].

2.8. In Vivo Effect of Piceatannol on Soild Tumor Growth and Metastasis. Male Donryu rats (3 weeks of age) were maintained on a stock pellet diet (CE-2, CLEA Japan, Tokyo, Japan) for 10 days. Starting 4 days prior to AH109A cell injections, the rats were fed AIN-93G basal diet containing 20\% casein (Oriental Yeast Co., Ltd., Tokyo, Japan), 39.75\% $\beta$ corn starch (Nihon Nosan Kogyo Co. Ltd., Yokohama, Japan), 13.2\% $\alpha$-corn starch (Nihon Nosan Kogyo Co. Ltd.), $10 \%$ sucrose (Nihon Nosan Kogyo Co. Ltd.), 7\% corn oil (Miyazawa Yakuhin Co. Ltd., Tokyo, Japan), 5\% cellulose powder (Oriental Yeast Co., Ltd.), 3.5\% mineral mixture 
(AIN-93G composition, Nihon Nosan Kogyo Co. Ltd.), 1\% vitamin mixture (AIN-93 composition, Nihon Nosan Kogyo Co. Ltd.), 0.3\% L-cystine (Wako Pure Chemical Industries, Ltd.), and $0.25 \%$ choline bitartrate (Wako Pure Chemical Industries, Ltd.). Piceatannnol was given to rats by adding $0.001 \%$ and $0.005 \%$ to the basal diet at the expense of $\beta$-corn starch. Each rat was implanted subcutaneously on one site in the dorsal region with $1 \times 10^{5} \mathrm{AH} 109 \mathrm{~A}$ cells suspended in PBS $(-)(0.5 \mathrm{~mL} / \mathrm{rat})$ to produce a solid tumor, as described previously [5]. The size of each solid tumor was measured every day. The tumor size is expressed as the sum of three measured dimensions, that is, height, length, and width. On day 20, the rats were deprived of their diets at 9:00 but allowed free access to water until they were sacrificed at 13:00. Solid tumors were dissected and weighed. Metastatic tumor foci in lung and inguinal and axillary lymphatic nodes were macroscopically examined and excised, and the number of metastatic foci was recorded.

2.9. Statistical Analysis. Data were expressed as means \pm SEM. Multiple comparison was performed by one-way analysis of variance (ANOVA) followed by Tukey-Kramer multiple comparisons test, and $P<0.05$ was considered statistically significant.

\section{Results}

3.1. Effect of Piceatannol on the Proliferation of Hepatoma Cells In Vitro and Ex Vivo. When added to medium, piceatannol dose-dependently and significantly reduced the proliferation of AH109A hepatoma cells. It commenced to decrease at $12.5 \mu \mathrm{M}$ and continued to decrease up to $200 \mu \mathrm{M}$ (Figure 1(A(a))). Of sera obtained from rats 1, 2, 3, and 6 hours after oral administration of piceatannol at a dose of $10 \mathrm{mg} / \mathrm{kg}$ body weight, serum obtained 2 hours after administration was found to show most prominent inhibitory effect against both the proliferation and invasion of AH109A hepatoma cells (data not shown). Thus, we prepared sera obtained from rats 2 hours after oral administration of piceatannol at doses $0,2.5,5$, and $10 \mathrm{mg} / \mathrm{kg}$ body weight. These sera also reduced dose-dependently and significantly the proliferation of AH109A cells as seen in Figure 1(A(b)).

3.2. Effect of Piceatannol on the Cell Cycle in Hepatoma Cells. When added to medium, piceatannol was demonstrated to induce cell cycle arrest at $\mathrm{G}_{2} / \mathrm{M}$ phase in $\mathrm{AH} 109 \mathrm{~A}$ cells at lower concentrations of 25 and $50 \mu \mathrm{M}$ (Figures $1(\mathrm{~B}(\mathrm{~b}))$ and $1(\mathrm{~B}(\mathrm{c})))$ as compared with the control $(0 \mu \mathrm{M})$ (Figure 1(B(a))).

3.3. Effect of Piceatannol on the Apoptosis Induction in Hepatoma Cells. In contrast to the effect on cell cycle, piceatannol failed to induce apoptosis at lower concentration of $50 \mu \mathrm{M}$ as compared with the control (Figures $1(\mathrm{C}(\mathrm{a})$ ) and $1(\mathrm{C}(\mathrm{b})))$. However, it induced strong apoptosis at higher concentration of $100 \mu \mathrm{M}$ (Figure $1(\mathrm{C}(\mathrm{c}))$ ).

3.4. Effect of Piceatannol on the Invasion of Hepatoma Cells In Vitro and Ex Vivo. When added to medium, piceatannol dose-dependently and significantly reduced the invasion of AH109A hepatoma cells. It commenced to decrease at $12.5 \mu \mathrm{M}$ and continued to decrease up to $200 \mu \mathrm{M}$ (Figure 2(A(a))). Sera obtained from rats 2 hours after oral administration of piceatannol at doses $0,2.5,5$, and $10 \mathrm{mg} / \mathrm{kg}$ body weight also reduced dose-dependently and significantly the invasion of AH109A cells as shown in Figure 2(A(b)).

3.5. Effect of Piceatannol on the Invasion of AH109A Cells Pretreated with Hydrogen Peroxide. To examine whether or not piceatannol would inhibit the invasion of tumor cells by its antioxidative activity, the invasion assay was performed with AH109A cells precultured in hydrogen peroxide- (ROS-) containing medium for 4 hours. As shown in Figure 2(B (a)), the invasive activity of AH109A cells precultured in ROS ( $25 \mu \mathrm{M}$ hydrogen peroxide) was significantly higher than that of AH109A cells with no treatment. Piceatannol suppressed the ROS-potentiated invasive activity of the precultured cells when added to the medium at a concentration of $10 \mu \mathrm{M}$ on preculturing the hepatoma cells with ROS. In our preliminary experiment, $12.5 \mu \mathrm{M}$ of piceatannol exposure for 4 hours to AH109A cells exerted no influence on the following AH109A proliferation during 24 hours (data not shown). Thus, the concentration of $10 \mu \mathrm{M}$ of piceatannol was adopted in this experiment to avoid an influence of the piceatannol-mediated proliferation inhibition on the AH109A invasion.

3.6. Intracellular Peroxide Levels of AH109A Cells Pretreated with Hydrogen Peroxide. As illustrated in Figure 2(B(b)), AH109A cells treated with $100 \mu \mathrm{M}$ hydrogen peroxide for 4 hours contained more intracellular peroxides than did control cells when analyzed with a flow cytometer using DCFH-DA as an indicator (control versus $\mathrm{H}_{2} \mathrm{O}_{2}$ ). Piceatannol $(10 \mu \mathrm{M})$ suppressed the rise in the intracellular peroxide levels of AH109A cells $\left(\mathrm{H}_{2} \mathrm{O}_{2}\right.$ versus $\mathrm{H}_{2} \mathrm{O}_{2}$ and piceatannol).

\subsection{Effect of Piceatannol on Sold Tumor Growth and Metastasis in Hepatoma-Bearing Rats. Dietary piceatannol (0.001\% and $0.005 \%$ ) tended to suppress the AH109A tumor size dose-dependently, although significant differences were not seen (Figure 3(A)). Accordingly, at the end of the 20-day treatment period, the weights of solid tumors were lower in the piceatannol-treated groups than in the control group (Figure 3(B)). The solid tumor weight of the $0.005 \%$ pi- ceatannol group was significantly reduced from $20.5 \pm$ 4.4 (control) to $9.4 \pm 2.5(0.005 \%$ piceatannol group $)$ $\mathrm{g} / \mathrm{rat}$, indicating that $c a .54 \%$ reduction was attained by $0.005 \%$ piceatannol. Numbers of metastatic foci were 0.22 , 0.2 , and 0 (number/rat) in the control, $0.001 \%$, and $0.005 \%$ piceatannol groups, respectively.}

\section{Discussion}

In the present study, piceatannol was demonstrated to inhibit both the proliferation and invasion of AH109A hepatoma cells in vitro. Sera obtained from Donryu rats orally given piceatannol also inhibited both the proliferation and invasion of AH109A hepatoma cells ex vivo. In our previous 

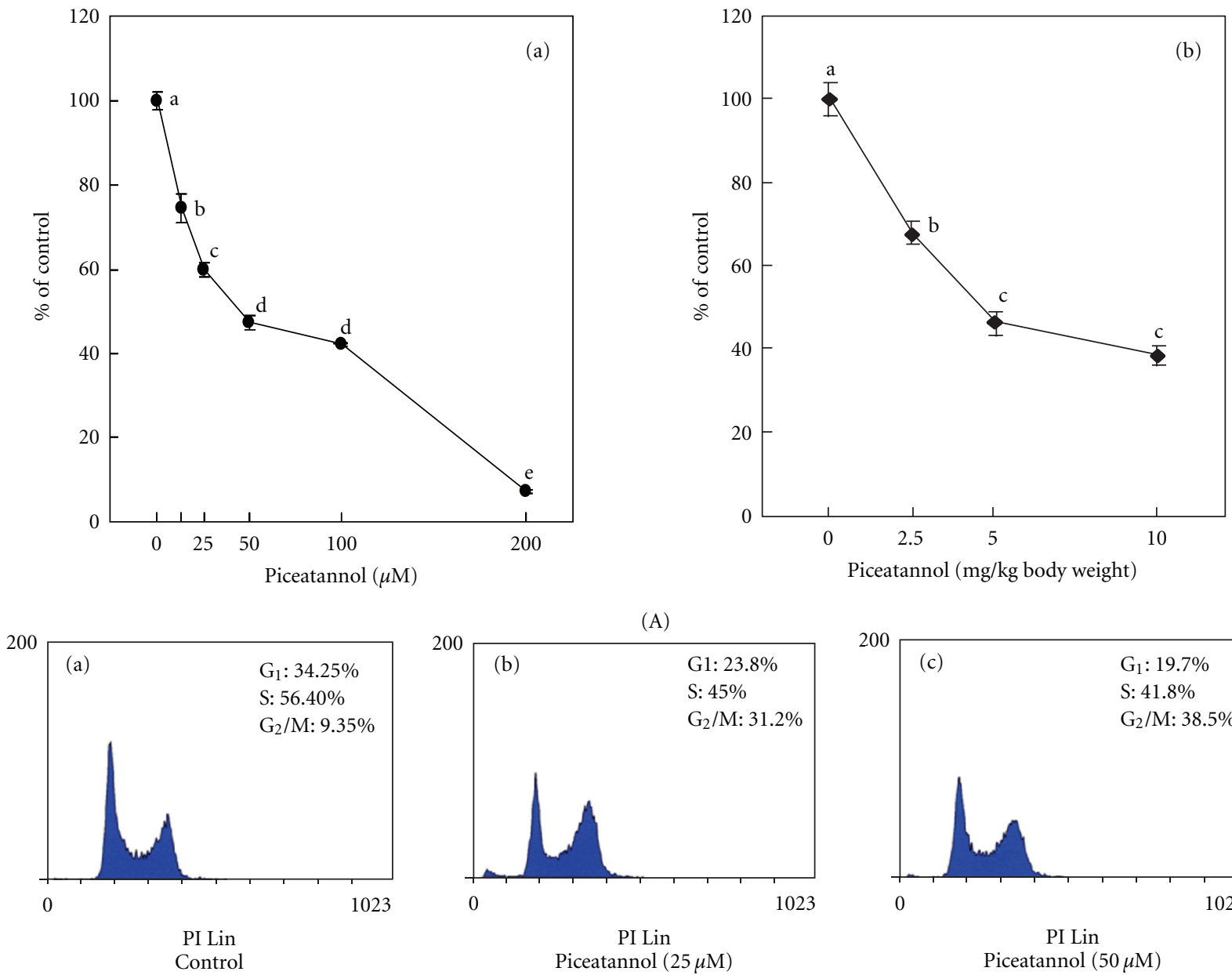

(A)
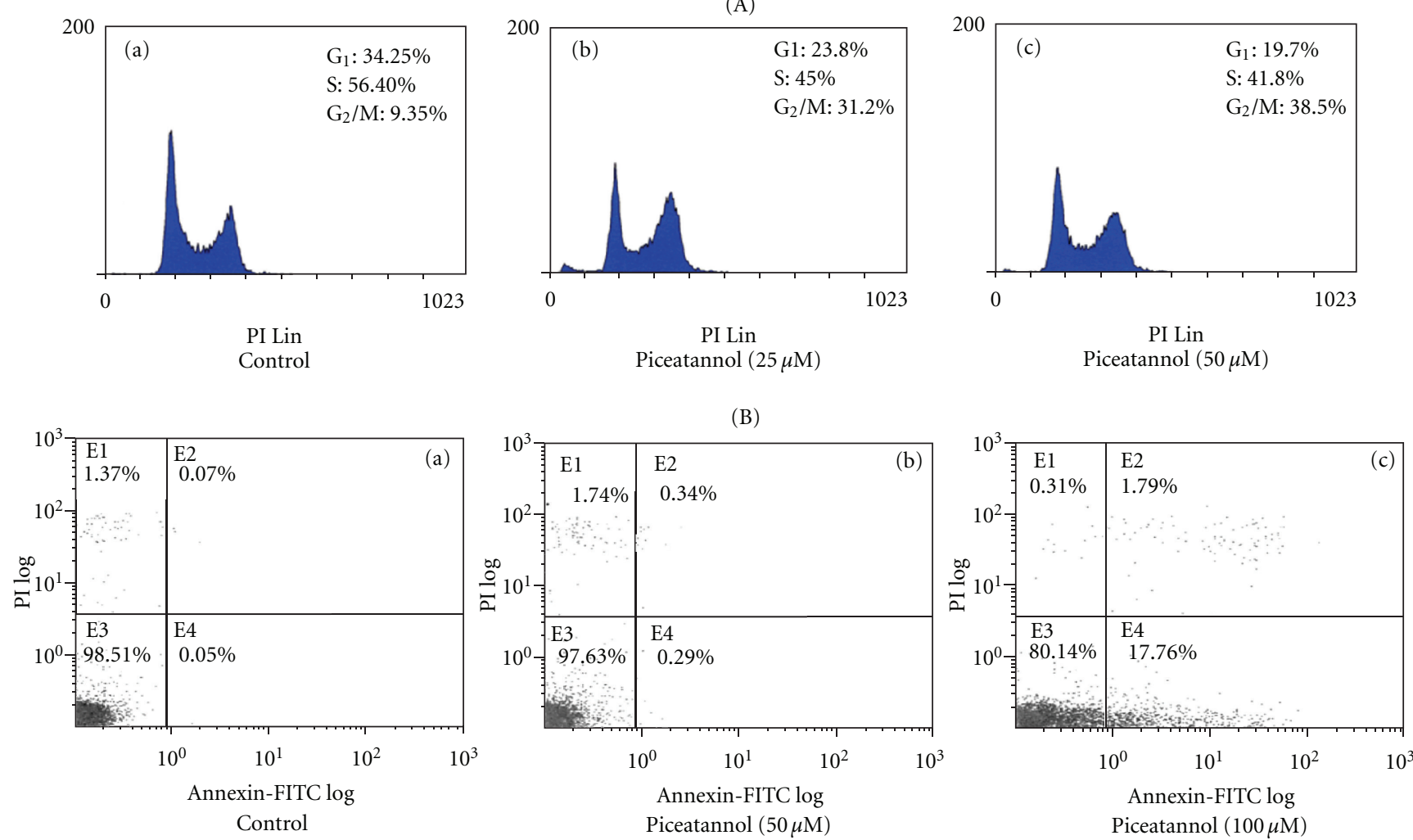

(C)

FIGURE 1: Effect of piceatannol on the proliferation (A), cell cycle (B), and apoptosis of AH109A hepatoma cells (C). (A) Piceatannol was dissolved in ethanol. The piceatannol solution was added to the culture medium at a final ethanol concentration of $0.2 \%$. Piceatannol was suspended in a $0.3 \%$ carboxymethyl cellulose sodium salt (CMC) aqueous solution at concentrations of $0.25,0.5$, and $1 \mathrm{mg} / \mathrm{mL}$. Piceatannol suspension or $0.3 \% \mathrm{CMC}$ alone (vehicle control) $(1 \mathrm{~mL} / 100 \mathrm{~g}$ body weight) was intubated to male Donryu rats which had been fasted overnight, and blood was collected $2 \mathrm{~h}$ after oral administration. The proliferative activity was determined by [methyl- $\left.{ }^{3} \mathrm{H}\right]$ thymidine incorporation method in vitro $(\mathrm{A}(\mathrm{a}))$ and ex vivo $(\mathrm{A}(\mathrm{b}))$. Each value represents the mean \pm SEM of six wells. Values not sharing a common letter are significantly different at $P<0.05$. (B) For cell cycle analysis, $3.0 \times 10^{5}$ cells of AH109A per well were seeded in the medium containing 0,25 , and $50 \mu \mathrm{M}$ piceatannol and cultured for $24 \mathrm{~h}$. Cells were then collected and washed twice with PBS(-). Thereafter, propidium iodide (PI) solution was added and cells were incubated for $30 \mathrm{~min}$ on ice. Cells at different cell cycle phases were then analyzed with a flow cytometer. Data are from representative experiment repeated three times with similar results (B(a)-(c)). (C) The effect of piceatannol on apoptosis in AH109A cells was assessed using Annexin V-FITC kit according to the manufacturer's instructions. Briefly, $5 \times 10^{5}$ cells of AH109A per well were seeded in a 6-well plate and cultured in the medium containing 0,50 , and $100 \mu \mathrm{M}$ piceatannol for $3 \mathrm{~h}$. At the end of culture, cells were labeled with Annexin-V-FITC and analyzed with a flow cytometer. Data are from representative experiment repeated three times with similar results $(\mathrm{C}(\mathrm{a})-(\mathrm{c}))$. 

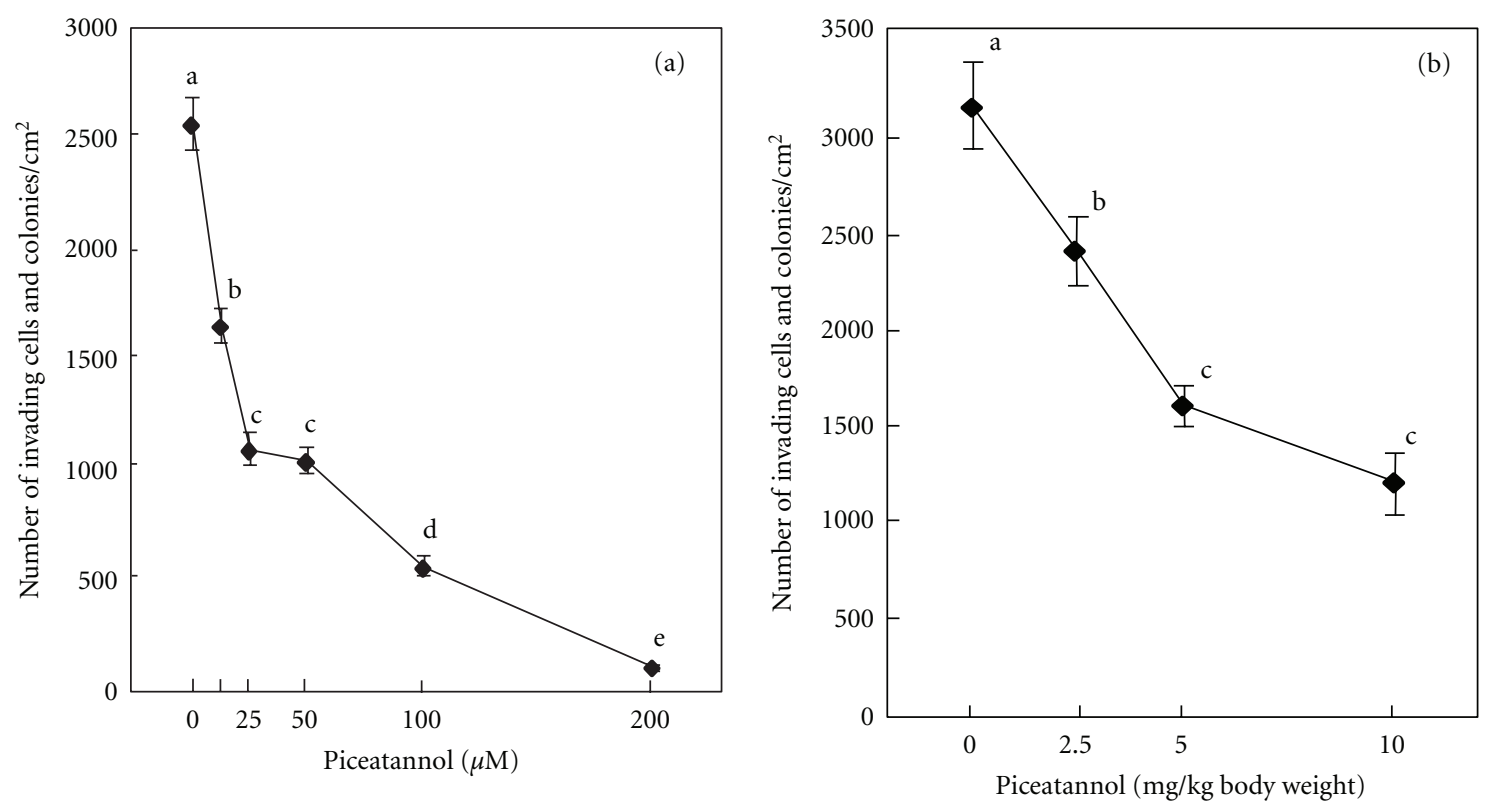

(A)
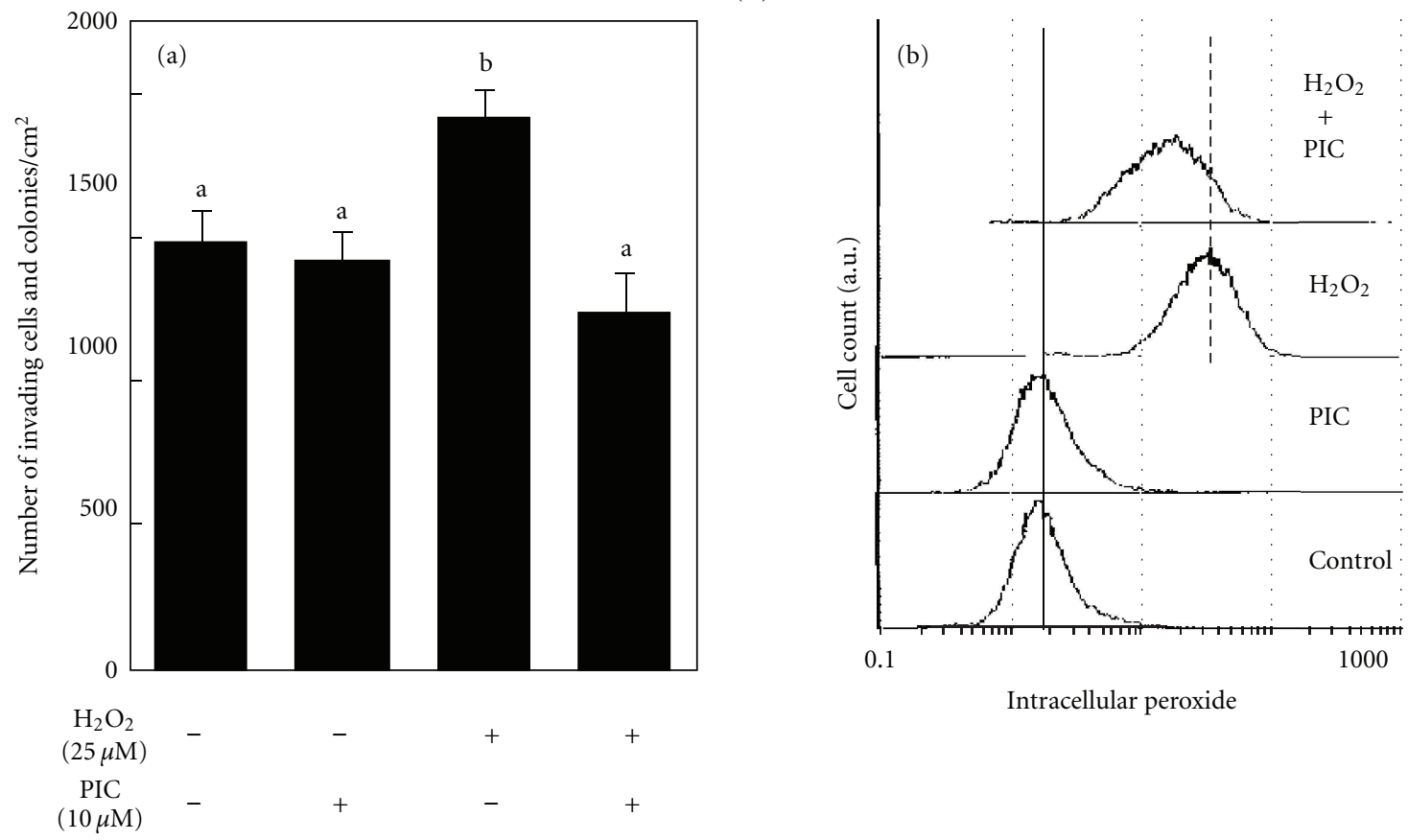

(B)

FIGURE 2: Effect of piceatannol on the invasion of AH109A hepatoma cells (A), reactive oxygen species- (ROS-) potentiated invasive activity and intracellular peroxide levels (B). (A) Piceatannol was dissolved in ethanol. The piceatannol solution was added to the culture medium at a final ethanol concentration of $0.2 \%$. Piceatannol was suspended in a $0.3 \%$ carboxymethyl cellulose sodium salt (CMC) aqueous solution at concentrations of $0.25,0.5$, and $1 \mathrm{mg} / \mathrm{mL}$. Piceatannol suspension or $0.3 \% \mathrm{CMC}$ alone (vehicle control) $(1 \mathrm{~mL} / 100 \mathrm{~g}$ body weight) was intubated to male Donryu rats which had been fasted overnight, and blood was collected $2 \mathrm{~h}$ after oral administration. The invasive activity was determined by AH109A-M cell coculture method in vitro (A(a)) and ex vivo (A(b)). Each value represents the mean \pm SEM of ten areas. Values not sharing a common letter are significantly different at $P<0.05$. (B) AH109A cells were cultured for $4 \mathrm{~h}$ in the absence or presence of $10 \mu \mathrm{M}$ piceatannol with or without $25 \mu \mathrm{M}$ hydrogen peroxide $\left(\mathrm{H}_{2} \mathrm{O}_{2}\right)$. AH109A cells were then washed once with $10 \% \mathrm{CS} / \mathrm{MEM}$ and seeded on the M-cell monolayer in 10\% CS/MEM without piceatannol and ROS. After cultured for $24 \mathrm{~h}$, invaded cells and colonies underneath M-cells were counted with a phase-contrast microscope. Each value represents the mean \pm SEM of ten areas. Values not sharing a common letter are significantly different at $P<0.05(\mathrm{~B}(\mathrm{a}))$. To evaluate the effect of piceatannol on intracellular peroxide levels, AH109A cells were treated with $100 \mu \mathrm{M} \mathrm{H}_{2} \mathrm{O}_{2}$ as ROS source. After ROS treatment for 1 hour, DCFH-DA was added, incubated for 20 minutes, and analyzed with a flow cytometer. Basal and ROS-potentiated intracellular peroxide levels are indicated as solid and dotted lines, respectively. Data are from representative experiment repeated three times with similar results $(\mathrm{B}(\mathrm{b}))$. 


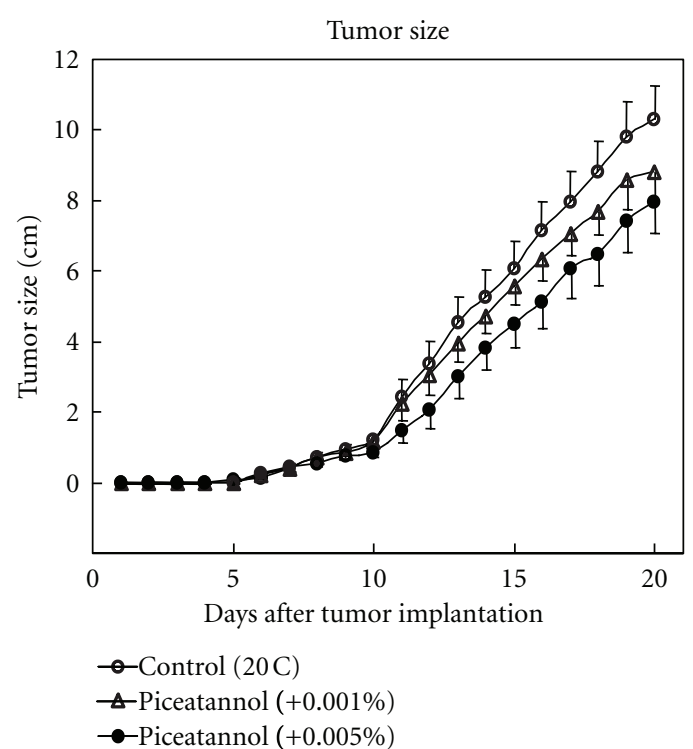

(A)

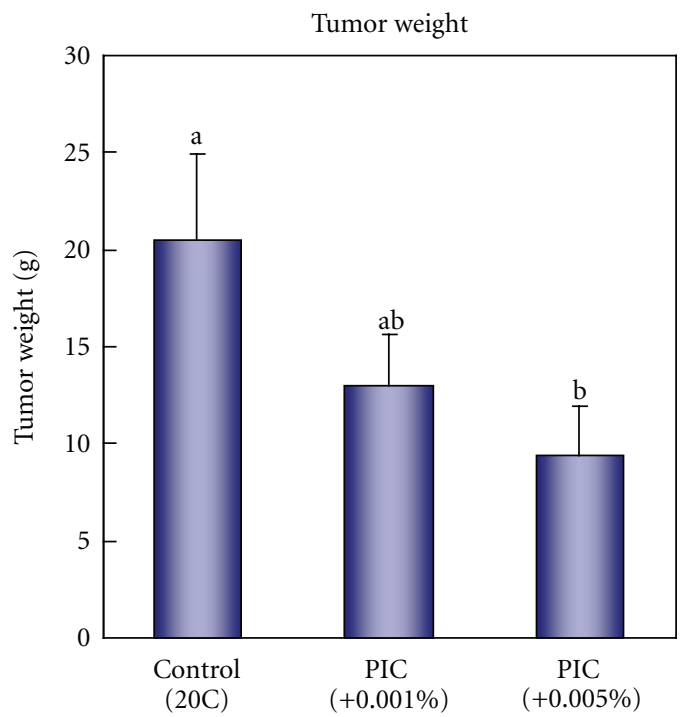

(B)

FIgURE 3: Effect of piceatannol on tumor size (A) and weight (B) of solid tumors in hepatoma-bearing rats. (A) Tumor size was determined in live animals by measuring the three-dimension sizes (height, length, and width). Each value represents the mean \pm SEM of nine (control and $+0.005 \%$ groups $)$ and ten $(+0.001 \%$ group) rats.

experiment, the sera obtained from Donryu rats orally given resveratrol completely lost the antiproliferative activity ex vivo, although its anti-invasive activity was preserved $[8,9]$. These previous and present findings suggest that metabolism of resveratrol by intestinal flora, intestine itself, or in the liver may be different from that of piceatannol. Precise reasons for the difference between two stilbenoids, however, are unclear at present.

To elucidate the mechanisms for the inhibition of hepatoma cell proliferation by piceatannol, the effect of piceatannol treatment on cell cycle and on the rate of apoptosis was examined by using flow cytometry. Piceatannol was found to suppress the proliferation from lower concentrations ( $25 \mu \mathrm{M}$ ) by arresting cell cycle of AH109A cells at $\mathrm{G}_{2} / \mathrm{M}$ phase (Figure 1(B)). Furthermore, piceatannol increased the rate of apoptosis at higher concentrations $(\sim 100 \mu \mathrm{M})$ (Figure $1(\mathrm{C})$ ). Dependencies of cell cycle arrest and apoptosis induction on piceatannol concentrations are similar to those on [6]gingerol [11]. Although the doses of piceatannol which induced apoptosis were higher than those which induced cell cycle arrest in AH109A hepatoma cells, these results suggest that piceatannol affects both cell proliferation and cell death which at least in part account for the inhibitory effect of piceatannol on the proliferation of AH109A cells.

Our previous studies have demonstrated that the invasion of AH109A cells is accelerated by ROS $[16,17]$. In the present study, we therefore examined the effect of piceatannol on the ROS-potentiated invasive activity using $\mathrm{H}_{2} \mathrm{O}_{2}$. Piceatannol was demonstrated to suppress the ROSinduced elevation of the AH109A invasion (Figure 2(B(a))). Piceatannol was also found to scavenge intracellular peroxide (Figure 2(B(b))). We have found that ROS can induce gene expression of hepatocyte growth factor (HGF), which is known as a cell motility factor [18], in M-cells as well as AH109A cells $[9,16]$. Thus, HGF produced by AH109A and M-cells may potentiate the motility of AH109A cells, leading to acceleration of the invasion of AH109A cells. If piceatannol, like resveratrol [9], suppresses the production of HGF through antioxidative activity, piceatannol may reduce the motility of AH109A invasion, this leading to the reduction of the AH109A invasion. Although preliminarily, we have measured HGF secretion from AH109A cells by ELISA. Piceatannol was found to dose-dependently suppress the HGF secretion after exposure of AH109A to piceatannol for 1 hour or 24 hours at the concentration ranges of $25-100 \mu \mathrm{M}$ (data not shown), suggesting an involvement of decreased HGF secretion in anti-invasive activity of piceatannol against $\mathrm{AH}-$ 109A hepatoma cells.

Although not significant, piceatannol dose-dependently suppressed solid tumor size in hepatoma-bearing rats (Figure 3(A)). However, solid tumor weights, a most simple but reliable index for tumor growth, were significantly reduced by feeding the $0.005 \%$ diet (Figure $3(\mathrm{~B})$ ). In addition to in vitro efficacy, piceatannol is demonstrated to be effective in vivo in suppressing tumor growth. Antimetastatic effect of piceatannol was not clear in vivo in the present study, this being probably due to weak metastatic activity in the control group. Selection and use of highly metastatic AH109A cell clone will clarify the in vivo antimetastatic effect of piceatannol.

In summary, piceatannol was clearly demonstrated to suppress both the proliferation and invasion of AH109A hepatoma cells in vitro and ex vivo. Antiproliferative effect might be due to induction of cell cycle arrest and apoptosis in AH109A, while anti-invasive effect might be due to antioxidative property of piceatannol. As was expected, piceatannol 
is shown to significantly suppress the solid tumor growth in vivo. These functions of piceatannol may be of significance from the aspects of both nutritional and pharmacological control of cancers.

\section{Acknowledgment}

This work was supported by the grant to KY from the Uehara Memorial Foundation, Tokyo, Japan.

\section{References}

[1] L. A. Liotta, U. Wewer, N. C. Rao et al., "Biochemical mechanisms of tumor invasion and metastases," Progress in Clinical and Biological Research, vol. 256, pp. 3-16, 1988.

[2] G. Zhang, Y. Miura, and K. Yagasaki, "Effects of green, oolong and black teas and related components on the proliferation and invasion of hepatoma cells in culture," Cytotechnology, vol. 31, no. 1-2, pp. 37-44, 1999.

[3] G. Zhang, Y. Miura, and K. Yagasaki, "Induction of apoptosis and cell cycle arrest in cancer cells by in vivo metabolites of teas," Nutrition and Cancer, vol. 38, no. 2, pp. 265-273, 2000.

[4] G. Zhang, Y. Miura, and K. Yagasaki, "Suppression of adhesion and invasion of hepatoma cells in culture by tea compounds through antioxidative activity," Cancer Letters, vol. 159, no. 2, pp. 169-173, 2000.

[5] D. Miura, N. M. Saarinen, Y. Miura, R. Santti, and K. Yagasaki, "Hydroxymatairesinol and its mammalian metabolite enterolactone reduce the growth and metastasis of subcutaneous AH109A hepatomas in rats," Nutrition and Cancer, vol. 58, no. 1, pp. 49-59, 2007.

[6] M. Jang, L. Cai, G. O. Udeani et al., "Cancer chemopreventive activity of resveratrol, a natural product derived from grapes," Science, vol. 275, no. 5297, pp. 218-220, 1997.

[7] T. C. Hsieh, P. Burfeind, K. Laud et al., "Cell cycle effects and control of gene expression by resveratrol in human breast carcinoma cell lines with different metastatic potentials," International Journal of Oncology, vol. 15, no. 2, pp. 245-252, 1999.

[8] Y. Kozuki, Y. Miura, and K. Yagasaki, "Resveratrol suppresses hepatoma cell invasion independently of its anti-proliferative action," Cancer Letters, vol. 167, no. 2, pp. 151-156, 2001.

[9] D. Miura, Y. Miura, and K. Yagasaki, "Resveratrol inhibits hepatoma cell invasion by suppressing gene expression of hepatocyte growth factor via its reactive oxygen species-scavenging property," Clinical and Experimental Metastasis, vol. 21, no. 5, pp. 445-451, 2004.

[10] M. Larrosa, F. A. Tomás-Barberán, and J. C. Espín, “The grape and wine polyphenol piceatannol is a potent inducer of apoptosis in human SK-Mel-28 melanoma cells," European Journal of Nutrition, vol. 43, no. 5, pp. 275-284, 2004.

[11] S. Yagihashi, Y. Miura, and K. Yagasaki, "Inhibitory effect of gingerol on the proliferation and invasion of hepatoma cells in culture," Cytotechnology, vol. 57, no. 2, pp. 129-136, 2008.

[12] H. Akedo, K. Shinkai, M. Mukai et al., "Interaction of rat ascites hepatoma cells with cultured mesothelial cell layers: a model for tumor invasion," Cancer Research, vol. 46, no. 5, pp. 2416-2422, 1986.

[13] Y. Miura, H. Shiomi, F. Sakai, and K. Yagasaki, "Assay systems for screening food components that have anti-proliferative and anti-invasive activity to rat ascites hepatoma cells: in vitro and ex vivo effects of green tea extract," Cytotechnology, vol. 23, no. 1-3, pp. 127-132, 1997.
[14] Y. Miura, K. Ono, R. Okauchi, and K. Yagasaki, "Inhibitory effect of coffee on hepatoma proliferation and invasion in culture and on tumor growth, metastasis and abnormal lipoprotein profiles in hepatoma-bearing rats," Journal of Nutritional Science and Vitaminology, vol. 50, no. 1, pp. 38-44, 2004.

[15] D. A. Bass, J. W. Parce, L. R. Dechatelet, P. Szejda, M. C. Seeds, and M. Thomas, "Flow cytometric studies of oxidative product formation by neutrophils: a graded response to membrane stimulation," Journal of Immunology, vol. 130, no. 4, pp. 1910-1917, 1983.

[16] Y. Miura, Y. Kozuki, and K. Yagasaki, "Potentiation of invasive activity of hepatoma cells by reactive oxygen species is mediated by autocrine/paracrine loop of hepatocyte growth factor," Biochemical and Biophysical Research Communications, vol. 305, no. 1, pp. 160-165, 2003.

[17] Y. Kozuki, Y. Miura, and K. Yagasaki, "Inhibitory effects of carotenoids on the invasion of rat ascites hepatoma cells in culture," Cancer Letters, vol. 151, no. 1, pp. 111-115, 2000.

[18] C. Parr and W. G. Jiang, "Expression of hepatocyte growth factor/scatter factor, its activator, inhibitors and the c-Met receptor in human cancer cells," International Journal of Oncology, vol. 19, no. 4, pp. 857-863, 2001. 

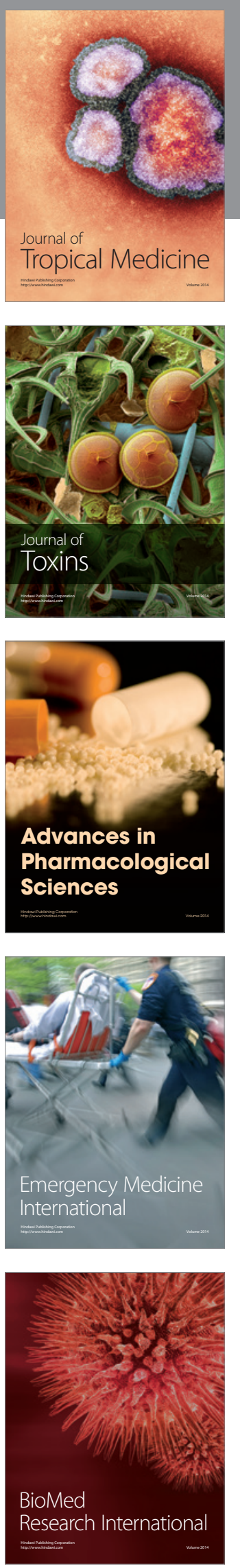
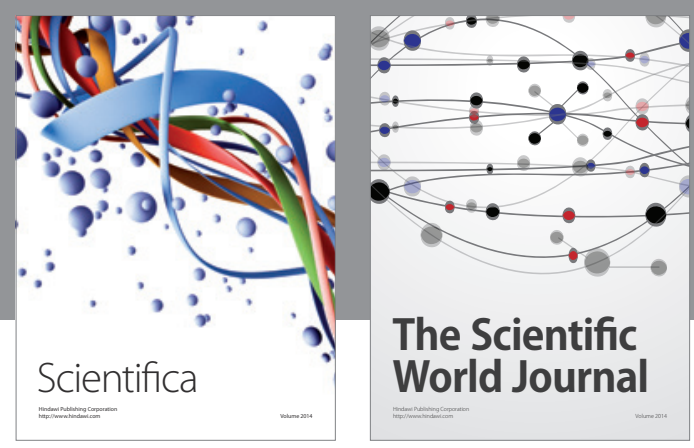

The Scientific World Journal
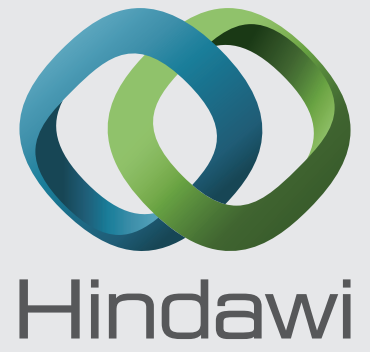

Submit your manuscripts at

http://www.hindawi.com
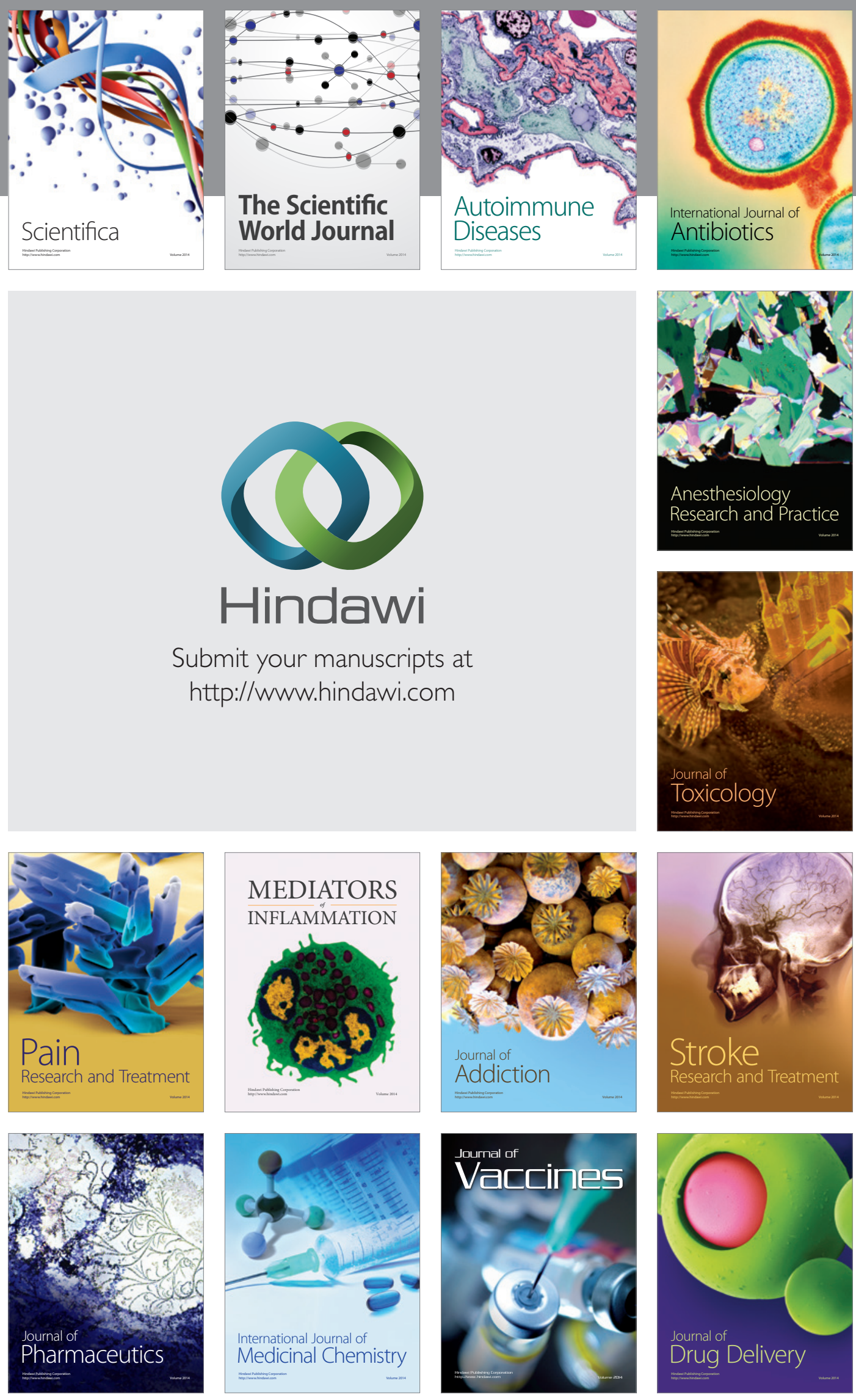